\title{
Desarrollo de un software para el modelamiento de las depositaciones inorgánicas incorporando daño a la formación por depositaciones inorgánicas en yacimientos de petróleo
}

\author{
Developing a software for the modeling of inorganic \\ deposition incorporating formation damage by inorganic \\ deposition in oilfields
}

\author{
Carmen Pinzón Torres ${ }^{1}$, Javier Felipe Ramirez ${ }^{2}$ y Luis Augusto Guanacas ${ }^{3}$
}

\begin{abstract}
Resumen
En este documento se describe el procedimiento realizado para el desarrollo de un software llamado ECOINCRUSTACIÓN cuya función es diagnosticar la reducción en la producción de petróleo debida a los depósitos inorgánicos en el yacimiento. En esta herramienta se incluyó un modelo termodinámico que permite predecir las condiciones a las cuales se formará precipitado de los componentes inorgánicos más comúnmente encontrados en sistemas de producción, tales como la calcita, barita, siderita, halita, hemidrita, yeso y anhidrita. El modelo se basa en la teoría de interacción iónica de Pitzer que permite representar matemáticamente la relación entre las diferentes especies iónicas presentes en la salmuera para luego utilizar otros modelos (Oddo y Tomson, 1991; Mogadashi et al, 2006; Valone y Skillern, 1982) y predecir la precipitación de sales poco solubles a diferentes condiciones físicas y químicas. Adicionalmente se desarrolló un modelo que describe el efecto de la incrustación inorgánica en el yacimiento a partir de los modelos de Robert (1996), Civan (2001) y Fadairo (2009), el cual se desarrolló con una modificación matemática propuesta por los autores; dicha modificación se realizó con el fin de ajustar el modelo a datos reales. El modelo físico de yacimiento calcula el daño a la formación, el radio de daño, la reducción en la permeabilidad y la caída de presión adicional por efecto de la depositación a partir de los resultados del modelamiento termodinámico de los minerales incluidos en el software.
\end{abstract}

Palabras clave: Modelo termodinámico de Oddo y Tomson; incrustación inorgánica, índice de saturación; masa de mineral precipitado; daño a la formación por depositaciones inorgánicas; modelo de daño a la formación.

\begin{abstract}
This document describes the procedure performed for the development of a software called ECOINCRUSTACIÓN whose function is to diagnosticate the reduction in oil production due to inorganic deposits on the reservoir. This tool provides a thermodynamic model that would predict the conditions under which there are going to precipítate inorganic compounds commonly found in production systems, such as calcite, barite, siderite, halite, hemidrita, gypsum and anhyrite. The model is based on the Pitzer's ionic interaction theory, allowing mathematically represent the relationship between the different ionic species present in the brine, and then use other models (Oddo and Tomson, 1991; Mogadashi et al, 2006; Valone and Skillern, 1982 ) and predict the precipitation of sparingly soluble salts with different physical and chemical conditions. In
\end{abstract}

1 - Qca. Esp en Educación Matemática, Universidad Surcolombiana, Neiva-Huila. carmen.pinzón@usco.edu.co

2- Ingeniero de Petróleos, Universidad Surcolombiana, Neiva-Huila.jfelipe.9209@gmail.com

3 - Ingeniero de Petróleos, Universidad Surcolombiana, Neiva-Huila.luis0494_my@hotmail.com 
addition, a model was developed to describe the effect of inorganic scale based on the reservoir models of Robert (1996), Civan (2001) and Fadairo (2009); and also it was developed with a mathematical modification proposed by the authors; such modification is made to fit the model to real data. The physical model calculates the reservoir formation damage, the radius of damage, the permeability reduction and additional pressure drop due to the deposition, from thermodynamic modeling results of minerals included in the software.

Keywords: Thermodynamic model; scale saturation, saturation index; precipitated mineral mass; formation damage by inorganic depositions; model formation damage

\section{Introducción}

La precipitación inorgánica es el fenómeno que se presenta por la sobresaturación de iones contenidos en una salmuera; en sistemas de producción el efecto de esta precipitación produce taponamiento de las líneas de flujo tanto superficiales como de subsuelo. Las caídas de presión, temperatura y cambios en las condiciones físicoquímicas del sistema facilitan la precipitación y depositación de sales (Moghadasi, 2003). La zona cercana a la cara del pozo comprendida por espacio poroso, sufre cambios notables en sus condiciones físicas como presión temperatura, y composición de los fluidos, que facilita la precipitación y depositación de minerales que taponan el espacio poroso y afectan la porosidad, la permeabilidad y finalmente la producción del pozo (Gruesbeck, 1982).

Las interacciones de los iones presentes en la salmuera son muy complejas y determinar su comportamiento en el yacimiento es aún más complicado debido al constante cambio de condiciones que se presentan en él debido a la producción. Pitzer (1973) propuso una teoría que modela las interacciones iónicas de una salmuera, y determina la participación de cada una en la formación de sales a diferentes condiciones de presión y temperatura. Con base en esta teoría se han desarrollado varios modelos termodinámicos para predecir las condiciones a la cuales se formarán los compuestos inorgánicos. Uno de los modelos más aceptado es el de Oddo y Tomson (1991), el cual se incluye en el software.

Los modelos termodinámicos ofrecen información importante sobre el estado de las depositaciones inorgánicas, sin embargo no cuantifican la severidad de su efecto en la formación. Debido a ésto se han desarrollado diferentes modelos de yacimiento; los primeros modelos se basaron en resultados experimentales, lo cual limitaba su aplicación solo al campo donde se realizaban los análisis de laboratorio (Gruesbeck, 1982 \& Wojtanowicz et al. 1987 \& Rochon et al. 1996). Posteriormente se diseñaron modelos analíticos que generalizan la aplicabilidad de sus ecuaciones para todos los campos; éstos por supuesto deben evaluarse particularmente para tratar de determinar la incertidumbre de sus parámetros y resultados, (Mogahadasi et al. 2006 \& Fadairo et al. 2009).

En este artículo se realiza una descripción de los principios utilizados para estudiar el fenómeno de precipitación inorgánica en sistemas de producción y además se desarrolla un modelo analítico para caracterizar el daño a la formación debido a la incrustación inorgánica, fundamentado en las relaciones existentes entre el volumen poroso ocupado por el mineral y su efecto sobre la dinámica de los fluidos dentro de estos poros.

Los modelos más representativos para la predicción y evaluación de las incrustaciones a nivel de formación se incluyen en el programa denominado "ECOINCRUSTACIÓN", el cual permite generar resultados de predicción de daño debido a la formación de incrustaciones inorgánicas, facilitando el proceso, ahorrando tiempo y costo en operaciones de tratamiento de pozos.

\section{Metodología}

\subsection{Modelamiento termodinámico para la predicción de incrustaciones.}

El modelo termodinámico incluido en el programa "ECOINCRUSTACIÓN" agrupa los principios de diferentes estudios para generar resultados más aproximados al fenómeno real de precipitación de sales. Dentro de los modelos más representativos utilizados se encuentran el modelo termodinámico de Oddo y Tomson (1991) y el modelo de Mogadashi et al (2003), en el cual se desarrolla un índice de saturación que determina el nivel de saturación de los iones presentes en la salmuera a condiciones de presión y temperatura específicas, basado en la composición electrolítica de la salmuera. Según el resultado de índice de saturación, se concluye la tendencia a 
precipitación de las diferentes sales, de esta forma:

Si IS $<0$ No existe tendencia a la precipitación

Si IS $=0$ El sistema se encuentra en equilibrio

Si IS $>0$ Existe tendencia a la precipitación

Para modelar el comportamiento de la solubilidad de las sales en la salmuera de producción se consideran parámetros como la constante de producto de solubilidad de cada uno de los minerales comúnmente encontrados en sistemas de producción de petróleo (calcita, barita, halita, hemidrita, anhidrita, yeso, celestita y siderita) y se tiene en cuenta el mecanismo de formación de incrustación, así como las condiciones que facilitan la precipitación de cada una de las sales. El modelo termodinámico incluye el efecto de la alta concentración de dióxido de carbono en la precipitación de los carbonatos y su relación con las propiedades fisicoquímicas de la salmuera.

La interacción entre los componentes iónicos de la salmuera a diferentes condiciones de temperatura y presión se evalúa por medio de la teoría de interacciones iónicas de Pitzer (1973). Esta teoría brinda el análisis necesario para evaluar las relaciones existentes entre los iones mediante el estudio de fuerzas iónicas de corto y largo alcance; la determinación de dichas fuerzas facilita calcular los coeficientes de actividad, que evalúan el grado de divergencia del comportamiento real de una solución electrolítica con respecto al comportamiento ideal.

El programa ECOINCRUSTACIÓN, calcula la masa de sal incrustada con base en el modelo termodinámico desarrollado por Valone y Skillern (1982), el cual retoma el estudio propuesto por Stiff y Davis (1952) para determinar cuantitativamente la sobresaturación de cada uno de los minerales. Para un mineral (MA), el equilibrio de solubilidad se expresa según la ecuación (1):

$$
M A_{(s)} \leftrightharpoons M_{(a c)}^{+}+A^{-}(a c)
$$

Si se genera precipitación del mineral, el producto iónico será mayor que la constante de producto solubilidad (Kps) de acuerdo con la ecuación (2):

$$
[m] *[a]>K p s
$$

Donde

$$
\begin{aligned}
& m: \text { Concentración inicial del ion } \boldsymbol{M}+(\mathrm{mol} / \mathrm{L}) \\
& a: \text { Concentración inicial del ion } \boldsymbol{A}-(\mathrm{mol} / \mathrm{L})
\end{aligned}
$$

La expresión de producto solubilidad después de la precipitación se representa en la ecuación (3):

$$
K p s=(m-Q)(a-Q)
$$

La expresión (3) es una ecuación cuadrática que se resuelve según la expresión (4):

$$
\bar{Q}=\frac{(m+a) \pm \sqrt{(m+a)^{2}-4(m a-K p s)}}{2}
$$

La ecuación (4) se utiliza para calcular las cantidades de precipitado para diferentes minerales.

El resultado generado por medio de la ecuación (4) se convierte en (lb/día) por medio de las conversiones respectivas (Ecuación 5). Esta conversión se realiza con el fin de evaluar la cantidad de masa depositada por día y así generar conclusiones y aplicar los procedimientos correctivos a nivel operacional.

\section{Masa Precipitada $=$ ppt ${ }^{*} V w^{*}$ Constante de precipitación}

Donde:

$V w=$ Volumen de Agua

$p p t=$ Moles de mineral que se precipita por kilogramo de agua.

La constante de precipitación es un factor de conversión que tiene en cuenta el caudal de agua producida por día.

Para el modelamiento termodinámico de la precipitación inorgánica debe tenerse información de las condiciones de operación del pozo y análisis fisicoquímicos del agua como presión, temperatura, composición iónica, alcalinidad, sólidos totales disueltos (TDS), $\mathrm{pH}$, fracción molar de dióxido de carbono y ácido sulfúrico y caudales de producción de agua petróleo y gas.

\subsection{Modelo de predicción del daño a la formación}

El aplicativo "ECOINCRUSTACIÓN" predice el efecto de la depositación inorgánica en la producción de petróleo con base en el modelo modificado de yacimiento de Fadairo (2009), que se expone en 2.21. Al modelo se le realizaron modificaciones, con el fin de deducir nuevas ecuaciones de daño y se compararon los resultados con el modelo original de Fadairo para analizar el grado de ajuste del modelo propuesto y el de Fadairo (2009), con datos reales. El desarrollo de la modificación del modelo se presenta a continuación: 
Desarrollo del modelo modificado de Fadairo et al.

Para el desarrollo del modelo se considera que los precipitados sólidos se suspenden uniformemente en un fluido incompresible $y$ en un medio poroso isotérmico, homogéneo e isotrópico; además, que la roca contiene un gran número de espacios porosos, los cuales están interconectados por la garganta de poro y que las fuerzas de interacción entre el medio y los minerales sólidos precipitados son insignificantes.

Se considera flujo radial de un fluido a una velocidad constante (q), saturado con partículas en estado sólido en una ubicación r, desde el "wellbore". Robert (1997) expresó el gradiente de presión debido al "scale" según la expresión (6):

$$
\frac{\mathrm{dp}}{\mathrm{dr}}=\frac{\mathrm{qB} \mu}{2 \pi \mathrm{K}_{\mathbf{0}} \mathrm{hr}_{\mathrm{s}} \mathrm{K}(\mathrm{C})}
$$

Donde $\mathrm{K}(\mathrm{C})$ es una función exponencial de daño a la formación:

$$
K(c)=e^{\left(-3 K_{d e p} C t\right)}
$$

Reemplazando la ecuación 7 en la ecuación 6 se obtiene una ecuación idealizada de flujo, expresada por Fadairo (2004), para calcular el gradiente de presión debido a la presencia de "scale" en la trayectoria de flujo (Ecuación 8):

$$
\frac{\mathrm{dp}}{\mathrm{dr}}=\frac{\mathrm{qB} \mu \mathrm{e}^{\left(3 \mathrm{~K}_{\mathrm{dep}} \mathrm{Ct}\right)}}{2 \pi \mathrm{K}_{\mathrm{o}} \mathrm{hr}_{\mathrm{s}}}
$$

Donde $\lambda_{k}$ evalúa el coeficiente de daño a la formación según la ecuación (9):

$$
\lambda_{\mathrm{k}}=\mathrm{e}^{\left(3 \mathrm{~K}_{\mathrm{dep}} \mathrm{Ct}\right)}
$$

La porosidad local instantánea se define como la diferencia entre la porosidad inicial y la fracción del espacio poroso ocupado por la incrustación (Ecuación 10):

$$
\phi_{\mathrm{s}}=\phi_{\mathrm{o}}-\phi_{\mathrm{d}}
$$

La ecuación (10) puede escribirse como:

$$
\phi_{\mathrm{s}}=\phi_{\mathrm{o}}-\frac{\text { Volumen de mineral depositado }}{\text { volumen total del espacio poroso }}
$$

El volumen de mineral (dV) que se forma y se deposita en el volumen del elemento en el intervalo de tiempo (dt) se describe con la ecuación (12):

$$
\mathrm{dV}=\mathrm{q}\left[\frac{\mathrm{dC}}{\rho \mathrm{dP}}\right]_{\mathrm{T}} \mathrm{dP} d t
$$

Siendo $\left[\frac{d C}{\rho d P}\right]_{T}$ el cambio de la concentración con respecto a la variación de presión a temperatura constante $\left(\mathrm{g} / \mathrm{m}^{3} \mathrm{~Pa}\right)$ y $\rho$ es la densidad de la incrustación formada $\left(\mathrm{g} / \mathrm{m}^{3}\right)$.

El cambio de la porosidad debido al daño se describe en la expresión (13):

$$
\mathrm{d} \phi_{\mathrm{d}}=\frac{\mathrm{q}\left[\frac{\mathrm{dC}}{\rho \mathrm{dP}}\right]_{\mathrm{T}} \mathrm{dP} \mathrm{dt}}{2 \pi \mathrm{K}_{\mathrm{o}} \mathrm{hr}_{\mathrm{s}} \mathrm{dr}}
$$

Sustituyendo la ecuación (8) en ecuación (13) se obtiene:

$$
d \phi_{d}=\frac{q\left[\frac{d C}{\rho d P}\right]_{T} d t}{2 \pi K_{o} h r_{s}} \frac{q B e^{\left(3 K_{d e p} C t\right)}}{2 \pi K_{o} h r_{s}}
$$

Integrando a ambos lados, considerando la variación del coeficiente de daño a la formación con el tiempo (principal diferencia con el modelo de Fadairo):

$\int_{0}^{\phi_{d}} d \phi_{d}=\frac{q\left[\frac{d C}{\rho d P}\right]_{T}}{2 \pi K_{o} h r_{s}} \frac{q B \mu}{2 \pi K_{o} h r_{s}} \int_{0}^{t} e^{\left(3 K_{d e p} C t\right)} d t$

Se obtiene la expresión (14):

$$
\phi_{d}=\frac{q^{2}\left[\frac{d C}{d P}\right]_{T} B \mu e^{\left(3 K_{d e p} C t\right)}}{4 \pi^{2} r_{s}^{2} h^{2} K_{o} \rho 3 K_{d e p} C}
$$

Sustituyendo la ecuación (14) en la ecuación (10) se obtiene la expresión para el cálculo de la porosidad instantánea, según la ecuación 15:

$$
\phi_{s}=\phi_{o}-\frac{q^{2}\left[\frac{d C}{d P}\right]_{T} B \mu e^{\left(3 K_{d e p} C t\right)}}{4 \pi^{2} r_{s}^{2} h^{2} K_{o} \rho 3 K_{d e p} C}
$$

Civan et al (1989), definen la porosidad instantánea como la relación entre la porosidad inicial y la función exponencial de daño a la formación (Ecuación 16):

$$
\phi_{s}=\phi_{o} e^{\left(-K_{d e p} C t\right)}
$$


Se define el cambio en la saturación de sal precipitada en un intervalo de tiempo (dt) como la relación entre el cambio de volumen de "scale" y el volumen poroso disponible durante dicho intervalo de tiempo según la expresión(17):

$$
\mathrm{dS}=\frac{\mathrm{q}\left[\frac{\mathrm{dC}}{\mathrm{dP}}\right]_{\mathrm{T}} \mathrm{dPdt}}{2 \pi \mathrm{hr}_{\mathrm{s}} \mathrm{dr} \phi_{\mathrm{o}} \mathrm{e}^{\left(-\mathrm{K}_{\mathrm{dep}} \mathrm{Ct}\right)}\left(1-\mathrm{S}_{\mathrm{w}}\right)}
$$

Sustituyendo la ecuación (8) en la ecuación (17) se obtiene la ecuación(18):

$\mathrm{dS}=\frac{\mathrm{q}\left[\frac{\mathrm{dC}}{\mathrm{dP}}\right]_{\mathrm{T}} \mathrm{dt}}{2 \pi \mathrm{hr}_{\mathrm{s}} \phi_{0} \mathrm{e}^{\left(-\mathrm{K}_{\mathrm{dep}} \mathrm{Ct}\right)}\left(1-\mathrm{S}_{\mathrm{w}}\right)} \frac{\mathrm{qB} \mu \mathrm{e}^{\left(3 \mathrm{~K}_{\mathrm{dep}} \mathrm{Ct}\right)}}{2 \pi \mathrm{K}_{0} \mathrm{hr}_{\mathrm{s}}}$

Integrando a ambos lados de la ecuación, se obtiene la expresión para calcular la fracción de mineral que ocupa el espacio poroso (19):

$$
S=\frac{q^{2}\left[\frac{d C}{d P}\right]_{T} B_{w} \mu_{w} e^{\left(4 K_{d e p} C t\right)}}{4 \pi^{2} r_{s}^{2} h^{2} K_{0} \phi_{o} \rho\left(1-S_{w i}\right) 4 K_{d e p} C}
$$

Esta ecuación se puede expresar de la siguiente forma:

$S=\frac{q^{2}\left[\frac{d C}{d P}\right]_{T} B_{w} \mu_{w} \lambda_{k}}{4 \pi^{2} r_{s}^{2} h^{2} K_{0} \phi_{o} \rho \lambda_{\phi}\left(1-S_{w i}\right) \frac{4}{3}\left(3 K_{d e p} C\right)}$

Reordenando la ecuación (20), se obtiene la ecuación (21):

$\frac{4}{3} \phi_{o} \lambda_{\phi} S\left(1-S_{w i}\right)=\frac{q^{2}\left[\frac{d C}{\rho d P}\right]_{T} B_{w} \mu_{w} t \lambda_{k}}{4 \pi^{2} r_{s}^{2} h^{2} K_{o} \rho}$

Sustituyendo la ecuación 21 en la ecuación 15 y dividiendo por $\phi_{o}$ se obtiene:

$$
\frac{\phi_{\mathrm{s}}}{\phi_{\mathrm{o}}}=1-\frac{4}{3} \lambda_{\phi} \mathrm{S}\left(1-\mathrm{S}_{\mathrm{wi}}\right)
$$

Expresando la permeabilidad inicial e instantánea en términos de la porosidad dañada (Civan et al., 1989; Frank et al., 1991), se obtiene la ecuación 23:

$$
\frac{\mathrm{K}_{\mathrm{s}}}{\mathrm{K}_{\mathrm{o}}}=\left(\frac{\phi_{\mathrm{s}}}{\phi_{\mathrm{o}}}\right)^{\mathrm{n}}
$$

Reemplazando la ecuación (22) en la ecuación (23), se obtiene la expresión (24) para calcular el daño a la permeabilidad por incrustación inorgánica:

$$
K_{s}=K_{o}\left[1-\frac{4}{3} \lambda_{\phi} S\left(1-S_{w i}\right)\right]^{n}
$$

\subsubsection{Factor daño y pérdida de presión adicional} debido a la depositación inorgánica

El factor de daño se expresa convencionalmente con la ecuación (25):

$$
\mathrm{s}=\left(\frac{\mathrm{K}_{0}}{\mathrm{~K}_{\mathrm{s}}}-1\right) \ln \frac{\mathrm{r}_{\mathrm{s}}}{\mathrm{r}_{\mathrm{w}}}
$$

Expresando la relación de permeabilidad en la ecuación (25) con la ecuación (24), se obtiene la expresión (26), con la cual se realiza el cálculo del factor de daño debido a la incrustación:

$s=\left(\left[1-\frac{4}{3} \lambda_{\phi} \operatorname{Ss}\left(1-S_{w i}\right)\right]^{-3}-1\right) \ln \frac{r_{s}}{r_{w}}$

Con base en la ecuación (26) y teniendo en cuenta la ecuación de Darcy para flujo radial, se deduce una expresión para el cálculo de la caída de presión adicional debido a la depositación inorgánica mostrada en la ecuación (27):

$\Delta \mathrm{P}_{\mathrm{s}}=\frac{\mathrm{qB} \mu}{2 \pi \mathrm{K}_{0} \mathrm{~h}\left[1-\frac{4}{3} \lambda_{\phi} \mathrm{S}_{\mathrm{s}}\left(1-\mathrm{S}_{\mathrm{wi}}\right)\right]^{3}} \ln \frac{\mathrm{r}_{\mathrm{s}}}{\mathrm{r}_{\mathrm{w}}}-\frac{\mathrm{qB} \mu}{2 \pi \mathrm{K}_{0} \mathrm{~h}} \ln \frac{\mathrm{r}}{\mathrm{r}_{\mathrm{w}}}$

Factorizando los términos comunes de la ecuación (27) se obtienen la expresión (28):

$\Delta \mathrm{P}_{\mathrm{s}}=\frac{\mathrm{qB} \mu}{2 \pi \mathrm{K}_{0} \mathrm{~h}}\left(\left[1-\frac{4}{3} \lambda_{\phi} \mathrm{S}_{\mathrm{s}}\left(1-\mathrm{S}_{\mathrm{wi}}\right)\right]^{-3}-1\right) \ln \frac{\mathrm{r}_{\mathrm{s}}}{\mathrm{r}_{\mathrm{w}}}$

Las ecuaciones (24), (26), (28) se utilizan para calcular parámetros relacionados con el efecto de la incrustación sobre la producción de petróleo. Estas ecuaciones se encuentran en el software "ECOINCRUSTACIÓN" y generan resultados con base en datos de propiedades físicas de roca y fluidos, y resultados del modelamiento termodinámico.

Tanto la disminución de presión adicional como factor de daño deben calcularse para cada mineral depositado, luego se suman los efectos de cada mineral sobre la permeabilidad, factor de daño y caídas de presión adicional, de acuerdo con las ecuaciones (29) a (31), donde $\mathrm{n}$ es el número de minerales depositados

$$
\begin{gathered}
K s_{\text {total }}=K o-\sum_{i=1}^{n} K s_{i} \\
S_{\text {total }}=\sum_{i=1}^{n} K s_{i} \\
\Delta P_{S_{\text {total }}}=\sum_{i=1}^{n} \Delta P_{S_{i}}
\end{gathered}
$$


Algunos datos de entrada al modelo, tales como $\left[\frac{d C}{\rho d P}\right]_{T}$ y $\mathrm{K}_{\mathrm{dep}} \quad$ son generados por el modelo termodinámico con base en la cantidad de masa precipitada y la tasa de precipitación, otros datos de entrada para el modelo físico de daño a la formación son el factor volumétrico $(\mathrm{Bw})$, viscosidad $(\mu \mathrm{w})$, saturación inicial de agua (Swi), porosidad ( permeabilidad promedio (Ko), espesor de la formación(h)y radio del pozo (rw).

\section{Resultados}

\subsection{Modelo termodinámico}

La validación del modelo termodinámico se realizó con datos proporcionados por una empresa operadora, comparando los resultados del software "ECOINCRUSTACIÓN" con los resultados de un software comercial "Scalesoft". Los resultados de índice de saturación calculados con base en un análisis fisicoquímico del año 2013, para unas condiciones de presión y temperatura específicas $\left(50\right.$ psi y $\left.160^{\circ} \mathrm{F}\right)$ se muestran en la figura 1 , donde se realiza una comparación cuantitativa entre el índice de saturación generado por cada uno de los aplicativos para cada mineral posiblemente precipitado.

\subsection{Modelo de yacimiento}

El modelo físico se aplicó para calcular los efectos de la depositación inorgánica a condiciones de yacimiento; los resultados fueron comparados con un estudio realizado por Franco et al (2012). En el estudio se muestra la reducción de la permeabilidad debida a la depositación de compuestos inorgánicos, especialmente de calcita, barita y siderita. Adicionalmente se relacionan los resultados con los obtenidos por el modelo de Fadairo (2009) para obtener una comparación entre los tres modelos aplicados para el estudio del daño a la formación. La semejanza entre los resultados obtenidos por el software ECOINCRUSTACIÓN, los resultados de Franco et al (2012) y el modelo original de Fadairo (2009) se muestra en la figura 2.

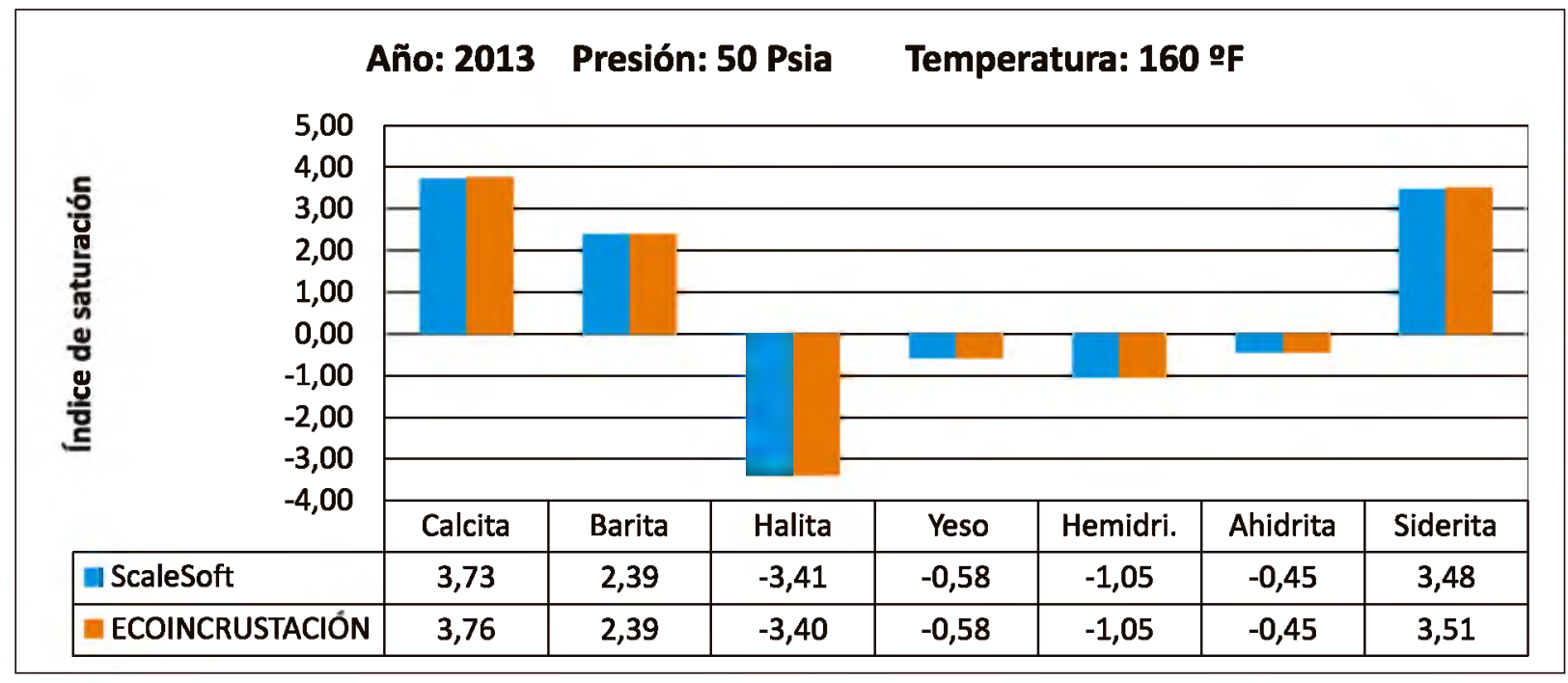

Figura 1. Comparación del índice de saturación obtenido con ScaleSoft y ECOINCRUSTACIÓN

Los resultados generados por el aplicativo ECOINCRUSTACION ajustan con los resultados obtenidos por Franco et al, esto se debe a la modificación realizada por los autores en el procedimiento matemático y la consideración de los cambios en la producción de agua, petróleo y gas, según la historia de producción de un pozo en particular. 


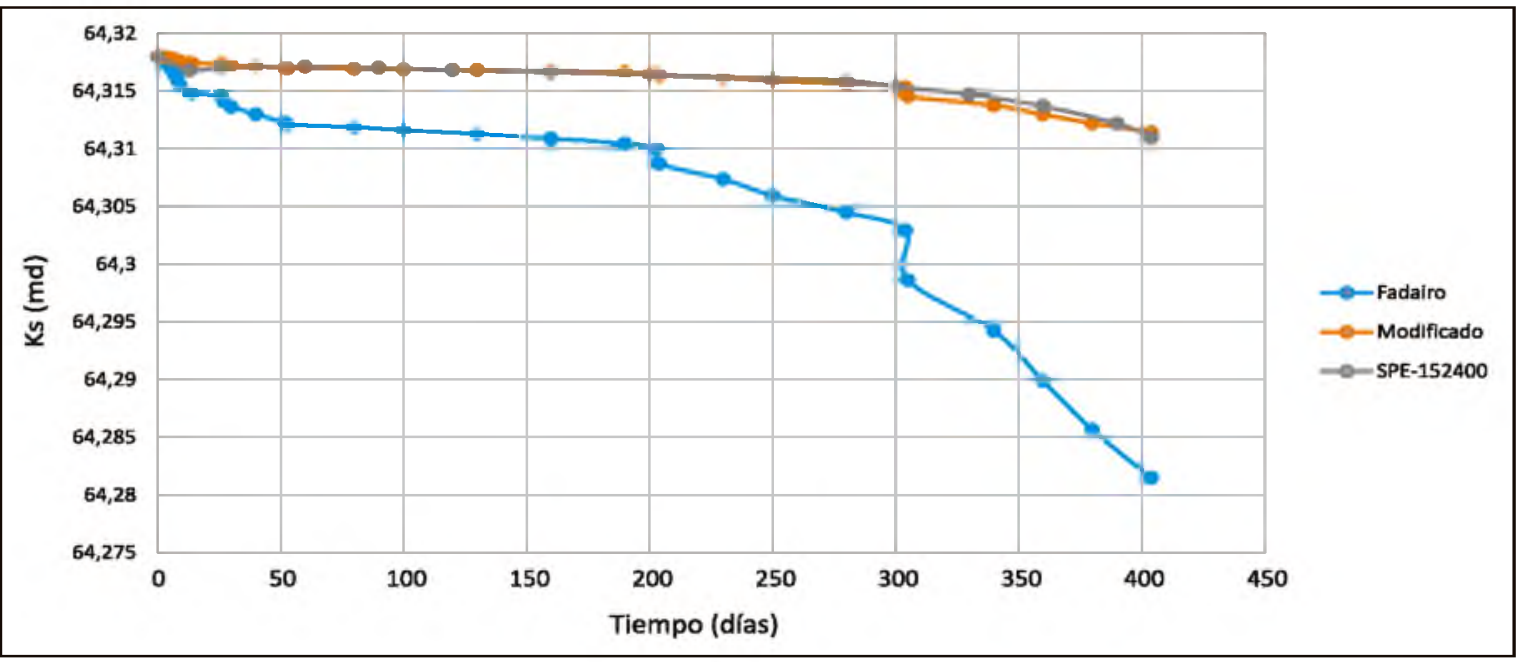

Figura 2. Gráfico de comparación del efecto de la depositación inorgánica sobre la permeabilidad
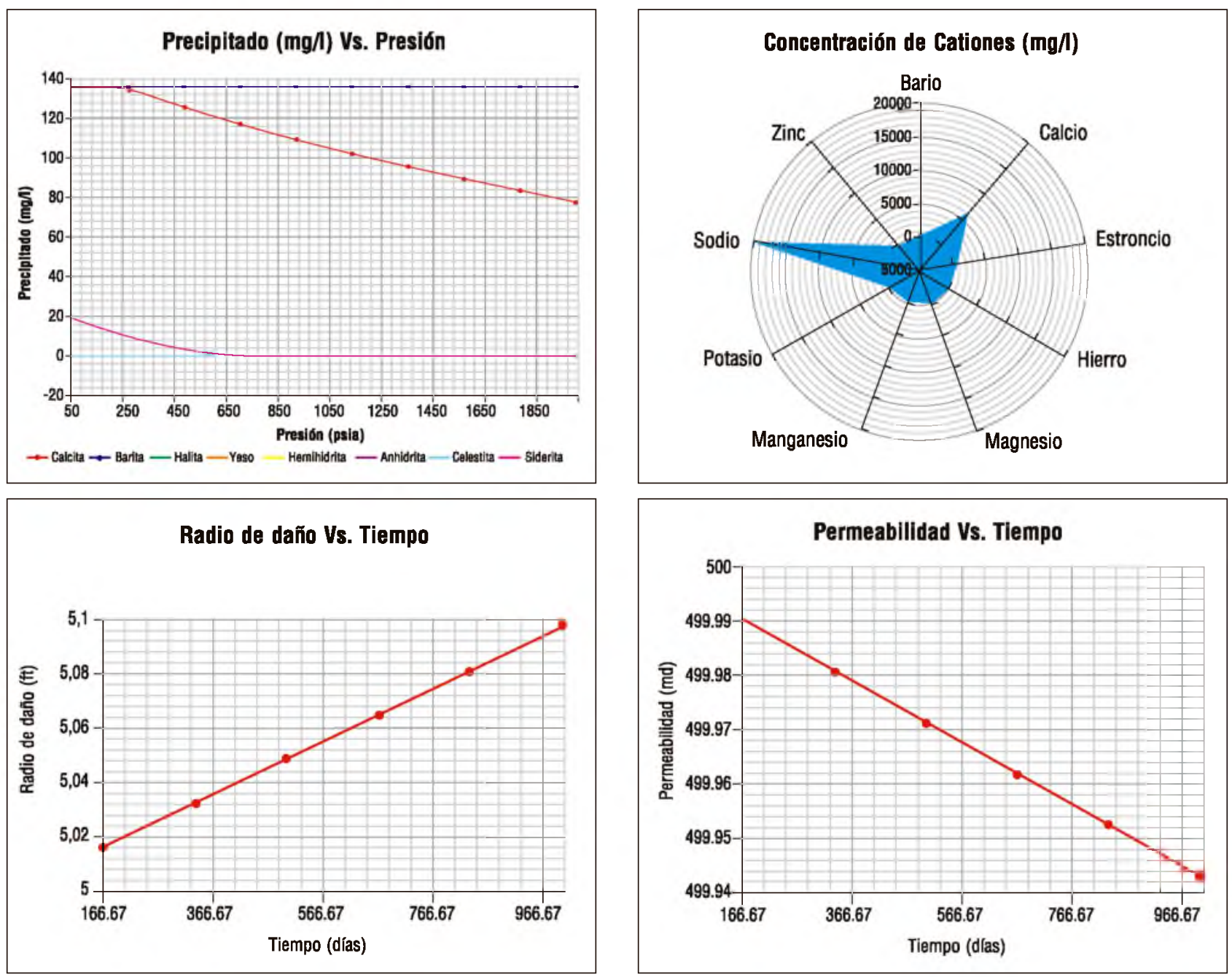

Figura 3. Gráficas de análisis del comportamiento y distribución de los iones y del cambio de presión y radio de daño con el tiempo 


\subsection{Generalidades del software}

\section{ECOINCRUSTACIÓN}

El programa presenta 3 módulos para calcular el índice de saturación y la masa depositada, estos módulos son el modelo químico, modelo termodinámico y modelo para mezclas de agua. Los dos últimos son los recomendados para la aplicación del modelo de yacimiento, ya que éstos consideran los parámetros termodinámicos del yacimiento como la presión y la temperatura. Sin embargo, si no se tiene un buen control de calidad de los datos de entrada al software no se pueden garantizar resultados confiables.

ECOINCRUSTACIÓN genera gráficas para ambos modelos (termodinámico y de yacimiento) que representan el comportamiento del índice de saturación, la cantidad de precipitado, la distribución radial de los iones, la expansión del radio de daño, la reducción de la permeabilidad y la caída de presión por efecto de la misma Figura 3.

\section{Conclusiones}

El modelamiento termodinámico utilizado para el estudio del índice de saturación y la masa precipitada, ajusta con otro software comercial para la mayoría de minerales aproximadamente en un $100 \%$. De manera similar, el modelo físico de yacimientos modificado mostró resultados más exactos que el modelo de Fadairo comparando ambos modelos con los resultados obtenidos por Franco et al (2012). Ésto se debe a que la formulación matemática del modelo modificado presentado por los autores del artículo no considera constante el coeficiente de daño a la formación y permite modelar la variación de los parámetros en el tiempo.

El daño a la formación por depósitos inorgánicos para el ejemplo de validación demostró ser insignificante para pozos productores, pero debe tenerse en cuenta su efecto en condiciones específicas de sistemas de inyección de agua, dada la complejidad que se genera por la mezcla de salmueras en el área cercana al pozo.

El uso del software ECOINCRUSTACIÓN favorece la predicción de la precipitación inorgánica y el efecto de la incrustación a nivel de yacimiento.

El software "ECOINCRUSTACIÓN" demostró ser una poderosa herramienta de diagnóstico, la cual genera resultados confiables siempre y cuando se realice el control de calidad de los datos de entrada.

\section{Referencias Bibliográficas}

1. Civan, F. Knapp, R. and Ohen, A., 1989. "Alteration of Permeability by fine Particle Processes". Vol. 3. SPE, Oklahoma City, OK.,pg. 65-79.

2. Civan, F., 2001. "Modeling Well Performance under Nonequilibrium Deposition Conditions". Paper SPE 67234 presented at the 2001 SPE Production and Operations Symposium held Oklahoma City, OK, 24-27.

3. Fadairo, A., 2004. "Prediction Scale Build up Rate around the Wellbore". M.Sc. Thesis Ibadan, Nigeria, University of Ibadan.

4. Fadairo, A., Omole, O. and Falode, O., 2009. "A Modified Model for Predicting Permeability Damage due to Oilfield Scale Deposition". Petroleum Science and Technology. Vol.27,pg. 1454-1465.

5. Franco, C.A., Garcia, C.C., Henao, W.A., Zapata, J.C., Ortiz, J.G., Bahamon, J.I. and Madera, K. 2012. "Formation Damage Modeling Improves Well Candidate Selection and Stimulation Treatment Design in Western Area of Putumayo Basin, Colombia". Paper SPE 152400 presented at the 2012 SPE Production and Operations Symposium held in Oklahoma City, OK, 24-27

6. Gruesbeck, C. and Collins, R.E., 1982. "Entrainment and Deposition of Fine Particles in Porous Media". Paper SPE 8430 presented at the 1982 SPE Annual Technical Conference and Exhibition held in Las Vegas, NV, 23-26.

7. Moghadasi, J., Jamialahmadi, M. and Mullersteinhagen, H., 2003. "Scale formation in Iranian Oil Reservoir and Production Equipment during Water Injection". Paper SPE 80406-MS presented at the 2003 International Oil Field Scale Symposium And Exhibition held in Aberdeen, UK, 29-30.

8. Mogadashi, J., Sharif, A., Kalantari, A. M. and Motiae, E., 2006. "A New Model to Describe Formation Damage during Particle Movement and Deposition in Porous Media". Paper SPE 99391 presented at the 2006 SPE Europec/EAGE annual conference and exhibition held in Vienna,AUT, 12-15.

9. Pitzer, K.S. 1973. "Thermodynamics of Electro-lytes. I. Theorical Basis and General Equations". Journal of Physical Chemistry, Vol. 77; No. 2 pg. 268-277.

10. Robert, B., 1997. "Effect of sulphur Deposition on Gas Well Performance". Paper SPE 36707.

11. Rochon, J., Creusot, M.R., Rivet, P., Roque, C. and Renard, M., 1996. "Water Quality for Water Injection Wells". Paper SPE 31122 presented at the SPE 1996 Formation Damage Control Symposium held in Lafayette, U.S.A., 14·15.

12. Stiff, H.A. and Davis, L.E., 1952. "A Method for Predicting the Tendency of Oilfield Waters to Deposit Calcium Carbonate". Paper SPE 952213-G.

13. Tomson, M.B. and Oddo, J.E., 1991. "A New Saturation Index Equation to Predict Calcite Formation in Gas an Oil Production". Paper SPE 22056-MS presented at the 1991 SPE International 
Symposium on Oilfield Chemistry held in Anaheim, California, CA, 20-22.

14. Valone, F.W. and Skillern, K.R., 1982. "An Improved Technique for Predicting the Severity of Calcium Carbonate". Paper SPE 10594-MS presented at the SPE 1982 International Symposium on Oilfield and Geothermal Chemistry, held in Dallas, TX, 25·27.

15. Wojtanowicz, A.K., Krilov, Z. and Langlinais, J.P., 1987."Study on the Effect of Pore Blocking Mechanisms on Formation Damage". Paper SPE 16233 presented at the SPE 1987 Production Operations Symposium held in Oklahoma City, OK. 8-10. 